\section{Electromagnetic Cavity Effects from Transmitters inside a Launch Vehicle Fairing}

Dawn H. Trout, Parveen F. Wahid, James E. Stanley

\begin{abstract}
:
This paper provides insight into the difficult analytical issue for launch vehicles and spacecraft that has applicability outside of the launch industry. Radiation from spacecraft or launch vehicle antennas located within enclosures in the launch vehicle generates an electromagnetic environment that is difficult to accurately predict. This paper discusses the test results of power levels produced by a transmitter within a representative scaled vehicle fairing model and provides preliminary modeling results at the low end of the frequency test range using a commercial tool. Initially, the walls of the fairing are aluminum and later, layered with materials to simulate acoustic blanketing structures that are typical in payload fairings. The effects of these blanketing materials on the power levels within the fairing are examined.
\end{abstract}

\section{Background:}

Intentional and inadvertent activation of spacecraft or vehicle emitters while inside a launch vehicle fairing is a longstanding issue in the space industry. The primary intentional $\mathrm{RF}$ emitters for spacecraft and launch vehicles operate at frequencies in the UHF, S, C and X Bands. It is often a requirement to evaluate the effects of spacecraft radio frequency (RF) transmission while enclosed within the payload fairing. Requirements for this evaluation can stem from a desire on the part of the spacecraft to transmit status signals to respective ground stations during launch, because of a desire not to change states while in the fairing, or due to insufficient inhibits to show that transmission will not occur. In addition, since at fairing separation launch vehicles typically change from external fairing antennas to internal antennas, there is often a transient RF cavity effect produced within the fairing until the fairing is completely deployed at separation. Because of short wavelengths and typically small antenna dimensions relative to spacing between emitters and sensitive equipment, analysis of the RF environment for the typical external antenna case can be performed in the far field under free space conditions thus making the calculations straight forward. In the enclosed fairing, however, multiple reflections, scattering and resonances occur that complicate this analysis. Since accurate modeling of these fields requires a mesh with no more than $1 / 10$ of a wavelength and fairing dimensions can be several meters, the computational capabilities required are enormous. With recent expansion in computational processing capability at $\mathrm{KSC}$ and the use of a Plexiglas fairing model used in a computational fluid dynamics modeling efforts in [1], a new study is underway to use actual test data to improve the ability to model this complicated cavity problem.

\section{Existing Data}

Separate testing and or analysis have been performed independently by launch vehicle providers. Moreover, this data is proprietary. Consequently, no consistent approach has been used across the launch vehicle community to deal with this cavity problem. This paper is using a generic test vehicle with generic materials that are typical of launch vehicles to evaluate this issue in a publishable forum.

Hallet and Redell [2] sought to quantify these fields using Poynting's theorem to solve for an equivalent wave that would dissipate the transmitted power in the surface areas of the surrounding fairing, blanketing materials and associated apertures ("the power supplied by the transmitter is equal to the power leaving through a closed surface."

The equation below is used to calculate the field [2]

$$
|E|=\sqrt{\frac{P_{t}}{2 \sum_{n=1}^{n=k} A_{n} \cdot \operatorname{real}\left(\frac{\eta_{n}}{\left|\eta_{0}+\eta_{n}\right|^{2}}\right)}}=\sqrt{\frac{P_{t}}{\frac{1}{2} \sum_{n=1}^{n=k} \operatorname{real}\left(\frac{4 A_{n} \cdot \eta_{n}}{\left|\eta_{0}+\eta_{n}\right|^{2}}\right)}}
$$

where

$\eta_{0}$ is the intrinsic impedance of the media (air) in the fairing volume.

$\eta_{n}$ is the impedance of the surface area exposed to the media 0 (air).

$A_{n}$ is the surface area in square meters.

$n$ is a designator for each surface type.

A scaled fairing model as well as an empty cavity was used to validate this method with some reasonable correlation. The actual test data reported in this reference was limited due to the proprietary nature of the study. The author's caution "this technique yields an assumed uniform field strength, not an exact solution of the field distribution." Significant modeling of equivalent complex impedances of typical fairing materials was performed for the study. This modeling has shown promise, but since spacecraft are expensive with often delicate instruments, it is necessary to have a more precise method of predicting the RF environment as testing to higher levels without adequate proof that these levels exist near these sensitive instruments is extremely unpopular.

One significant contribution of this report was the technique of accounting for layered materials. Commercial tools that our group has evaluated that are capable of performing computational electromagnetic analysis of this scale do not allow specification of layers at a boundary. This report used equivalent impedances to allow the entire layered boundary to be represented as one equivalent boundary material. This technique will be utilized with the commercial tool and presented in future publications to evaluate the blanketed test case presented here. The prediction method and to a lesser extent the test data shows a standing wave effect when layered 
blanketing is used at specific frequencies where the distance between the layers with some conductivity approach multiples of one quarter of a wavelength.

\section{Test Configuration:}

The test article shown in Figure 1 used for this testing was a Plexiglas enclosure in the shape of a representative fairing bolted at the edges and supported by a metal frame. The internal cavity was lined with industry aluminum foil and smoothed as much as possible to simulate aluminum fairing walls of a typical launch vehicle and the perfect electric conductor of the computer model.

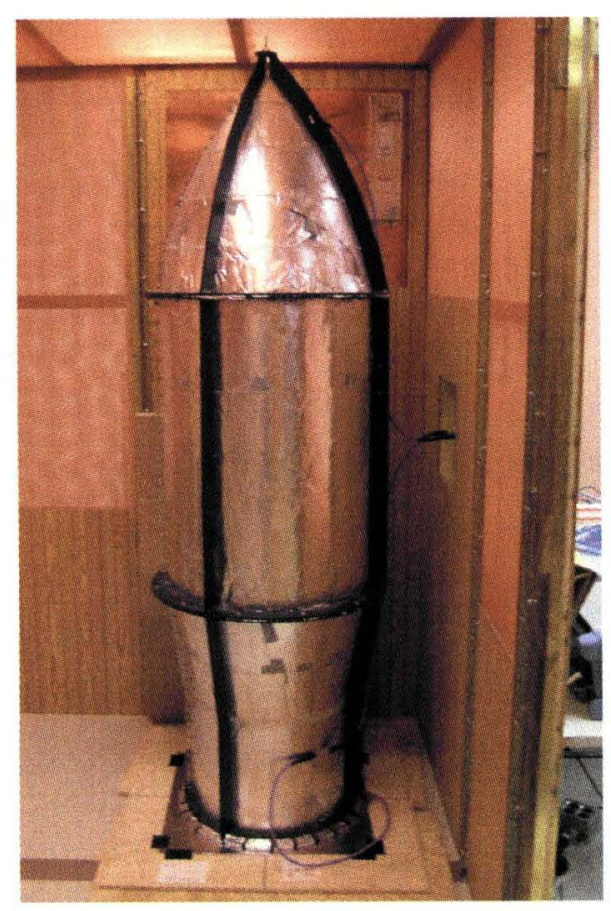

Figure 1. Scaled Fairing Plexiglas Model with Aluminum Foil

Baseline tests were performed in this configuration before other lining materials were added. A low input level of zero (0) $\mathrm{dBm}$ or one milliWatt $(1 \mathrm{~mW})$ from an Agilent E8257D Signal Generator was used for most of the test, although an amplifier was used to generate higher levels to verify that the power level chosen would not affect the results. This data showed linear correlation with power input increase therefore only the $0 \mathrm{dBm}$ data will be presented here. A Spectrum Analyzer was used to measure the received power from the pick-up antenna. This set-up is shown in Figure 2.

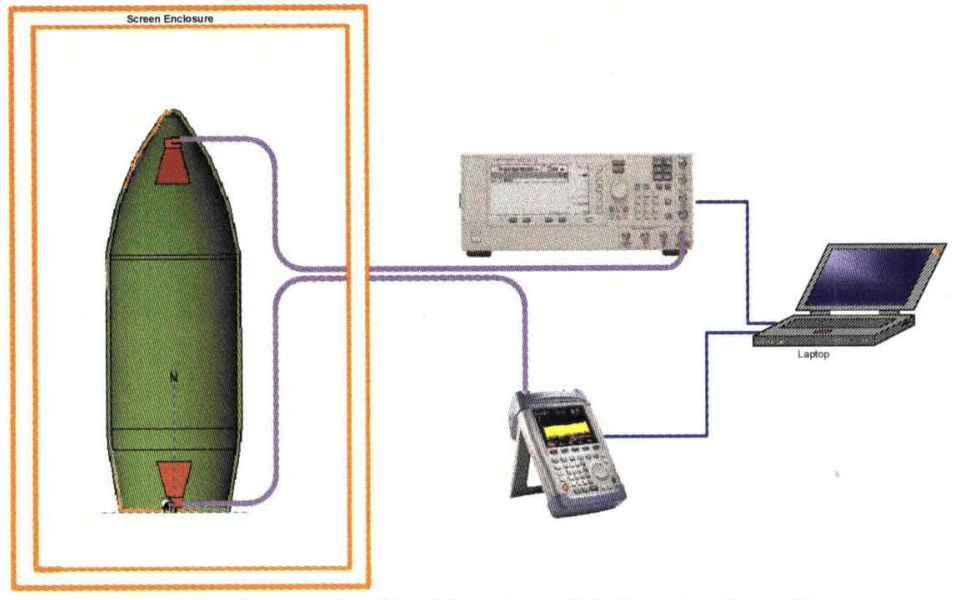

Figure 2. Pro_E model of half-cut on fairing to show Test Set-up

The EMCO 3115 double ridge guide horn antenna used for both transmit and receive antennas at the top and bottom of the test fairing is shown in Figure 3.

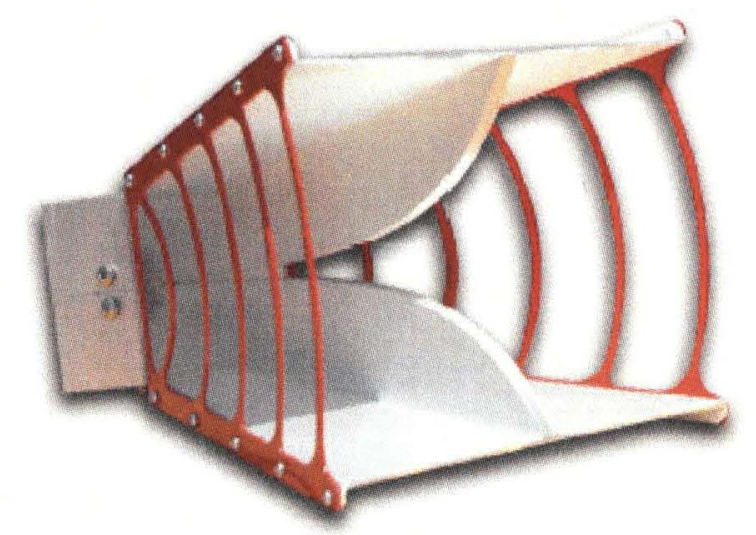

Figure 3. EMCO 3115 Double Ridge Guide Horn Test Results

Data showing the frequency response of multiple power levels in the aluminum lined mock fairing configuration is given in Figure 4. Although there is variation across the $750 \mathrm{MHz}$ to $18 \mathrm{GHz}$ Frequency range, the data shows that these measurements are scalable with power level. 


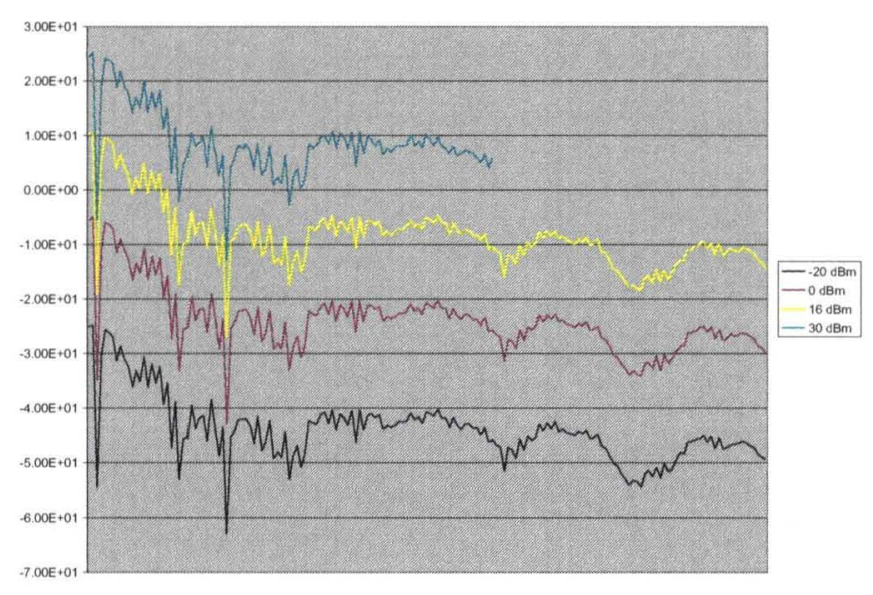

Figure 4: Power Measurements with Bare Aluminum at varied power levels.

Kapton $377^{\circledR}$ manufactured by DuPont was used as a liner for the cavity since this material is specifically designed for RF absorption and Kapton is a material frequently used for launch vehicle and spacecraft blanketing materials for its thermal insulating properties. Standard 1" foam was used to separate the blanketing material. The test cases were studied for walls (i)Aluminum (ii) Aluminum-Kapton (iii) Aluminum-KaptonFoam, and (iv)Aluminum-Kapton-Foam-Kapton. Data was recorded for each test case from $750 \mathrm{MHz}$ to $18 \mathrm{GHz}$ and shown in Figure 5.

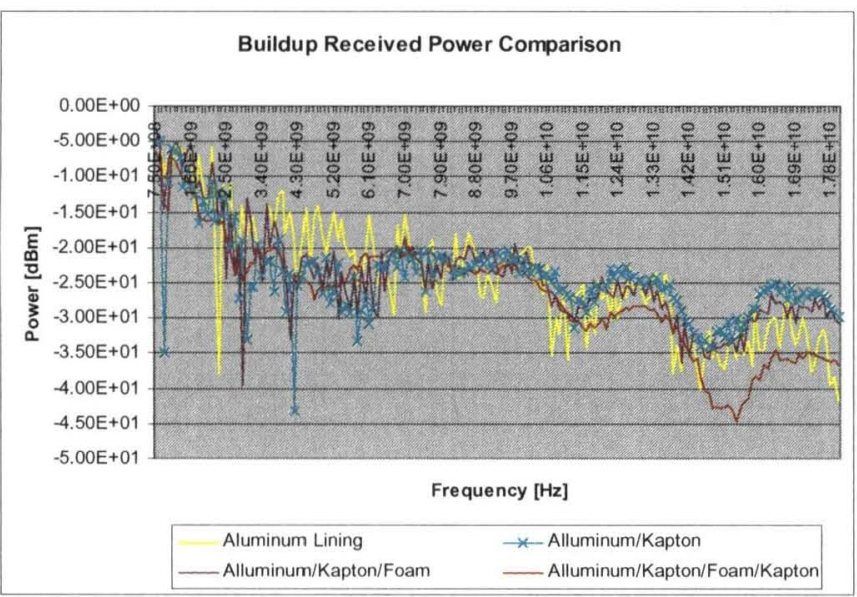

Figure 5: Power Measurements with Bare Aluminum and Various Blanketing Materials

The test data obtained for the aluminum lined fairing shows wide variation in power levels. This case has predominately the highest envelope except at frequencies in the upper range of the test data (above $14 \mathrm{GHz}$ ) where the addition of foam and Kapton resulted in slightly higher levels. In the $4-8$ $\mathrm{GHz}$ range the aluminum lined case is 10 to $20 \mathrm{~dB}$ above the power data from all other lined cases. Each additional layer caused less fluctuations in the power levels especially at higher frequencies. Minimal effects were observed from adding the foam only which is expected since the impedance of this material approaches free space. A profound smoothing effect was observed with the multiple layers of Kapton and an approximately $20 \mathrm{~dB}$ decrease with this layering above 14 $\mathrm{GHz}$.

Ref 2 also showed attenuation in field strengths with similar blanket materials from the bare fairing wall case by $8-26 \mathrm{~dB}$. Similar results were found here, however the standing wave pattern predicted from the separation between the layers of the blanketing materials was not nearly as pronounced as in Ref 2 .

\section{Modeling}

The test data collected is then compared to the simulation data generated by the electromagnetic simulation software, EM Software \& System's FEKO.

A Pro-E model of the plexiglass structure was developed and imported into FEKO. The fairing walls were modeled as perfect electric conductors in the FEKO model. The Antenna was built in FEKO to match the antenna structure of EMCO 3115 shown in Figure 3. The pattern from this model was then obtained as shown in Figure 7 and used in the modeled fairing structure.

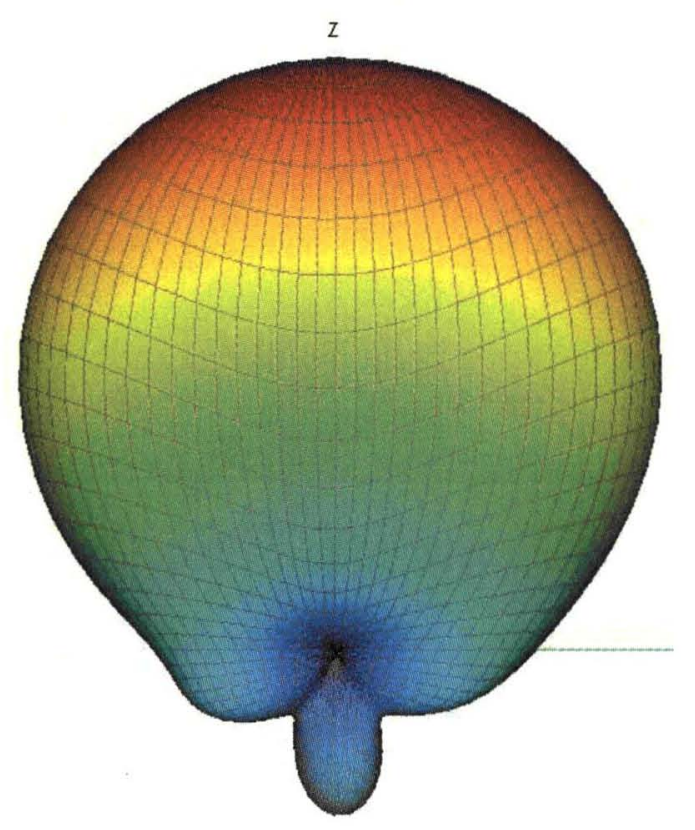

Figure 6. FEKO Model of EMCO 3115 Double Ridge 3-D

The results compared nicely with the published EMCO gain pattern data. One plane pattern is shown in Figure 7. 


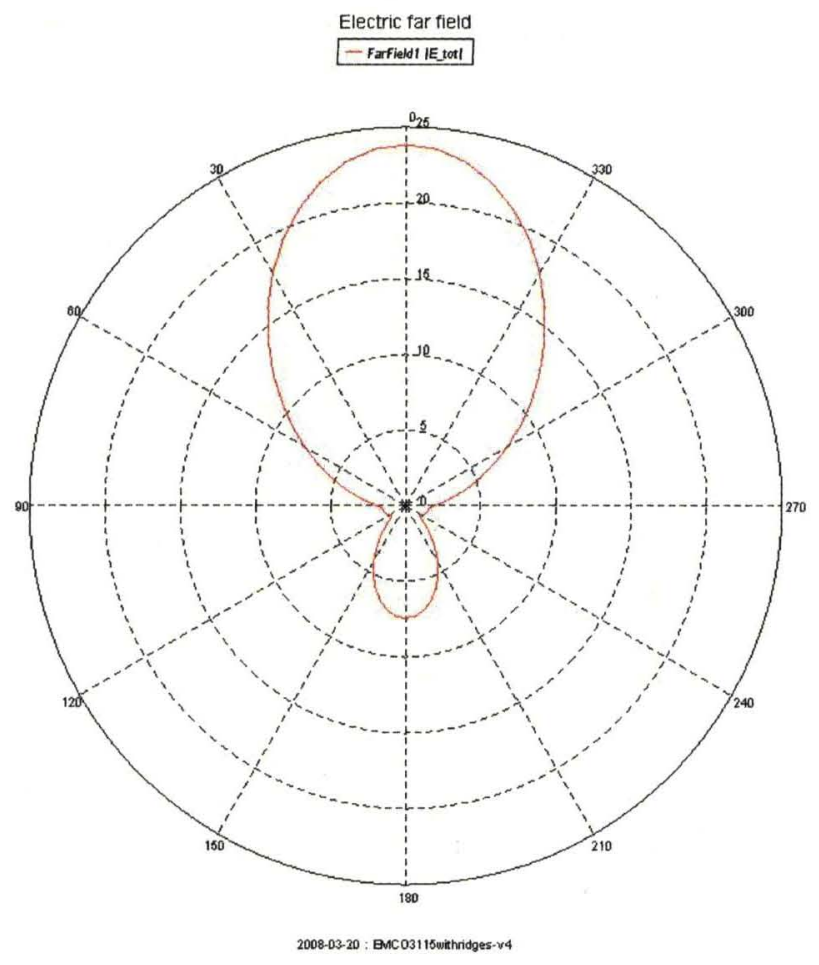

Figure 7. EMCO 3115 Double Ridge Plane Antenna Pattern

The bare fairing wall case was simulated at 1 to $1.9 \mathrm{GHz}$ in $100 \mathrm{MHz}$ increments using a hybrid Method of Moments/Physical Optics model.

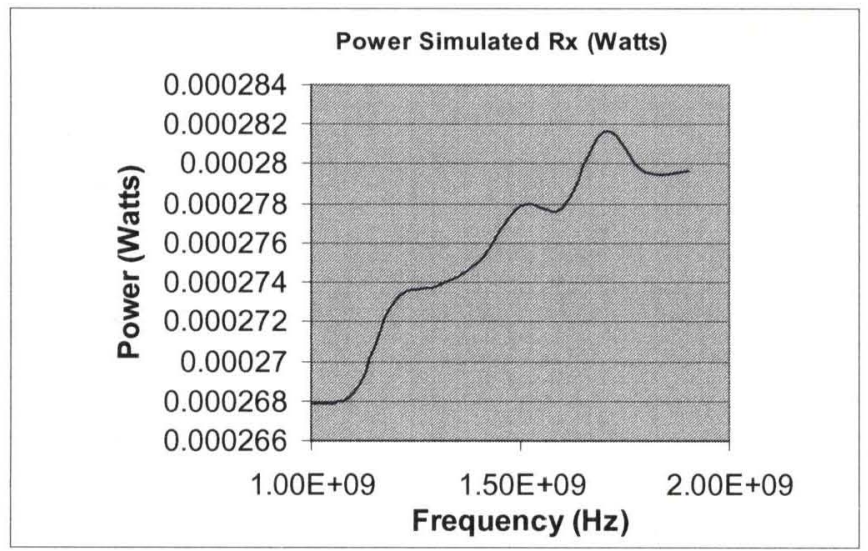

Figure 8: Simulated Received Power

Figure 8 shows received power from the simulated case. Although there is a general increase in the power received with frequency, the variation is only $0.22 \mathrm{~dB}$ over the range from 1.0 to $1.9 \mathrm{GHz}$. The power losses shown at particular frequencies in the test cases were duplicated in these results.

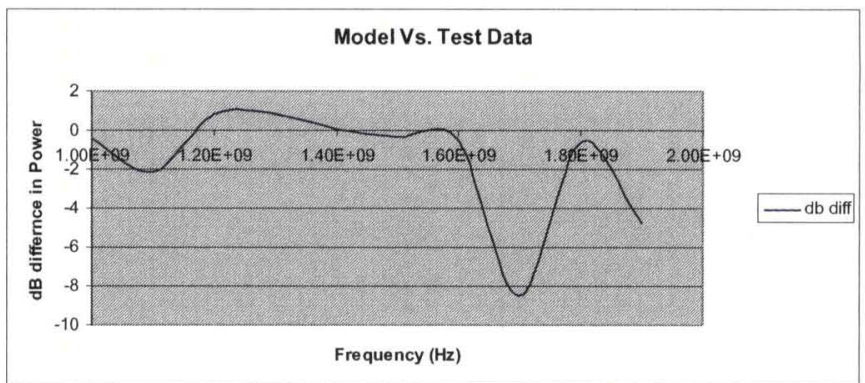

Figure 9: Differences between test data and model

The model predicts the peaks of test data within $1 \mathrm{~dB}$ when test data is adjusted for the typical high VSWR in the $1-2 \mathrm{GHz}$ range. The power losses in the cavity are not predicted in the model as accurately and the most significant loss at $1.7 \mathrm{GHz}$ test case was approximately $8 \mathrm{~dB}$ lower than the model case. Comparisons to the Free Space Case at $1 \mathbf{~ G H z}$

To evaluate the validity of the test results from a general sense, the free space predicted value was calculated. At 1 $\mathrm{GHz}$, using $2 \mathrm{D} / \lambda^{\wedge} 2$ criteria, this antenna would be in free space. Where $\mathrm{D}$ is the largest dimension of the antenna and $\lambda$ is the wavelength.

$\operatorname{Pr}=\operatorname{Pt} *(\lambda / 4 \pi r)^{\wedge} 2 *(G)^{\wedge} 2=(1 \mathrm{~mW})\left(0.3 /(4 \pi(1.57))^{\wedge} 2 *(4.09)^{\wedge} 2\right.$ $=3.8 \mu \mathrm{Watts}$

Where:

$\operatorname{Pr}=$ Power Received

$\mathrm{Pt}=$ Power Transmitted

$\mathrm{R}=$ distance between transmit and receive antennas in meters $\mathrm{G}=$ gain of the antenna at $1 \mathrm{GHz}$

The measured power at $1 \mathrm{GHz}$ was $203 \mu$ Watts. The difference between these values can be attributed to amplification from the cavity.

$10 * \log (203 / 3.8)=17.27 \mathrm{~dB}$

Comparing this value to an approximate Volume Q for PEC per reference 3, we obtain: (The approximate volume is $0.3632 \mathrm{~m}^{\wedge} 3$ )

$\mathrm{Q}=\left(16 \pi^{\wedge} 2 \mathrm{~V}\right) / \lambda^{\wedge} 3=2124=33 \mathrm{~dB}$

Accordingly, it is reasonable that the measured power levels within the cavity are significantly higher than the free space calculated values.

\section{Conclusions:}

This study shows how the fields increase in the fairing model lined with a good conductor, the effects of the various blanketing materials that typically line the fairing walls in launch vehicles, and the effects of adding a conductive spacecraft. Losses contributed to blanketing materials were 
shown to be comparable to the available industry reports.

Preliminary modeling results from 1.0 to $1.9 \mathrm{GHz}$ range

which showed peak levels were well matched.

The variations in the power data in the aluminum only case was expected due to multiple cavity reflections. The layered $\mathrm{RF}$ absorbing liners test cases had the biggest reduction on the observed power levels. Although there was variation with frequency, no pronounced standing wave pattern appeared as with Ref 2 data.

\section{Future Work:}

Testing should be modified to examine more points in the cavity to identify changes in eigenmodes especially with the addition of a spacecraft model. Reference 4 shows multiple examples of various eigenmodes for a conical structure. An omni-directional antenna will also be used to remove uncertainties regarding increases in field from the gain of the antenna. Further investigation into the differences in power absorption modeled versus test case is warranted as well as an extension of the modeled frequency range and cases considered.

\section{References:}

1. "CFD Validation with LDV Test Data for Payload/Fairing Internal Flow", AIAA, Kandula, Hammad, Schallhorn

2. Technique for Predicting the RF Field Strength Inside and Enclosure, M. Hallett, J. Redell, NASA/TP - 1998-206864

3. D. A. Hill, M. T. Ma, A. R. Ondrejka, B. F. Riddle, M. L. Crawford, and R. T. Jonk, "Aperture excitation of electrically large, lossy cavities,” IEEE Trans. Electromagn. Compat., vol. 36, no. 3, pp. 169-177, Aug. 1994.

4. "Resonant Modes of Conical Cavity", Greg Egan, Website www.gregegan.net/Science/Cavity/Cavity.html. 